\title{
Comparative Study between Metformin and Laparoscopic Ovarian Drilling for Ovulation Induction, Achievement of Pregnancy and Live Birth in Asian Women with Polycystic Ovarian Syndrome: A Prospective Trial
}

\author{
Samsad Jahan ${ }^{*}$, Samira Humaira Habib², Md. Sakandar Hyet Khan ${ }^{3}$, Akter Jahan4, \\ Reefat Nayer ${ }^{1}$, Farzana Sharmin ${ }^{5}$ \\ ${ }^{1}$ Department of Gynecology \& Obstetrics, BIRDEM, Dhaka, Bangladesh \\ ${ }^{2}$ Health Economics Unit, BADAS, Dhaka, Bangladesh \\ ${ }^{3}$ Refugee Health Unit, MOHFW, Cox's Bazar, Bangladesh \\ ${ }^{4}$ Govt. Homeopathic College, Dhaka, Bangladesh \\ ${ }^{5}$ Department of Obstetrics and Gynecology, BIHS, Dhaka, Bangladesh \\ Email: shelly_birdem@yahoo.com, dhcdp@dab-bd.org, samirahumaira@yahoo.com
}

Received 21 April 2014; revised 6 June 2014; accepted 16 July 2014

Copyright (C) 2014 by authors and OALib.

This work is licensed under the Creative Commons Attribution International License (CC BY).

http://creativecommons.org/licenses/by/4.0/

(c) (i) Open Access

\section{Abstract}

Objective: To compare the efficacy of metformin and laparoscopic ovarian drilling to be used in anovulatory patients with polycystic ovarian syndrome (PCOS) for ovulation induction and pregnancy achievement. Materials and Methods: Setting and Design: A prospective clinical trial conducted in the outpatient department of Gynaecology and Obstetrics of BIRDEM hospital from August 2008 to August 2013. Patient(s): Three hundred and eight newly diagnosed patients with PCOS based on ESHRE/ASRM criteria. These patients were assigned to two groups: Group 1 (152 patients) received $500 \mathrm{mg}$ of metformin three times a day; Group 2 (156 patients) received laparoscopic ovarian drilling. Main Outcome Measure(s): Rate of ovulation, pregnancy rate (PR), and live birth. Ovulation was monitored by serum progesterone, transvaginal sonography up to six cycles or till pregnancy occurred upto six months. Result(s): The ovulation rate was $68.4 \%$ in the metformin group, $59 \%$ in the laparoscopy treatment group. The pregnancy rate was $(20.1 \%$ and $16.6 \%)$ and live birth rate was $(18.4 \%$ and $15.4 \%)$ in metformin and laparoscopy treatment groups, respectively. The rate of spontaneous first trimester loss was three and two in metformin and laparoscopy group respectively. There were no ectopic pregnancies in metformin group and two in laparoscopy group. There was no second trimester pregnancy loss in metformin group, three in laparoscopy group. There were no multiple pregnancies in metformin group, two in lapa-

\footnotetext{
${ }^{*}$ Corresponding author.
}

How to cite this paper: Jahan, S., et al. (2014) Comparative Study between Metformin and Laparoscopic Ovarian Drilling for Ovulation Induction, Achievement of Pregnancy and Live Birth in Asian Women with Polycystic Ovarian Syndrome: A Prospective Trial. Open Access Library Journal, 1: e645. http://dx.doi.org/10.4236/oalib.1100645 
roscopy group.

\title{
Keywords
}

\author{
Polycystic Ovarian Syndrome, Metformin, Laparoscopy
}

\author{
Subject Areas: Global Health, Gynecology \& Obstetrics
}

\section{Introduction}

Infertility has been attributed to various factors, amongst which anovulation is the cause of about $40 \%$ of all female infertility. Polycystic ovary syndrome (PCOS) is one of the most common endocrinopathies affecting 5\% $10 \%$ of reproductive age women [1]. It was originally described as a triad consisting of obesity, hirsutism and oligomenorrhoea, infertility [2]. This condition is a potential health hazard with long-term sequelae, notably type 2 diabetes, cardiovascular disease and hyperlipidemia [3]. An internationally accepted definition has been adopted in 2003 by the European Society for Human Reproduction and Embryology and the American Society for Reproductive Medicine, known as the ESHRE/ASRM Rotterdam consensus [4]. It required the presence of two of the following three diagnostic criteria: 1) oligomenorrhea or anovulation; 2) clinical or biochemical evidence of hyperandrogenism and 3) the presence of polycystic ovarian morphology. Women suffering from PCOS have low fecundity with anovulation [5] and to an extent, early pregnancy loss [6] and pregnancy complications [7]. Induction of ovulation would restore ovulation and pregnancy. For many years clomiphene citrate (CC) has been the standard treatment for ovulation induction for these patients. Women who fail to ovulate or get pregnant with CC usually were recommended for ovarian diathermy surgery, gonadotropin treatment or even IVF. However, these treatments are expensive and associated with side effects [8]. Because hyperinsulinemia may contribute to hyperandrogenism and infertility in women with PCOS, agents that can ameliorate insulin resistance and reduce circulating insulin level can be used [9] [10]. The most extensively studied insulin-lowering agent in the treatment of PCOS was metformin.

Metformin is a biguanide oral antihyperglycemic agent, which has been extensively used in the treatment of type 2 diabetes mellitus. It lowers blood glucose levels mainly by inhibiting hepatic glucose production and increases in the peripheral glucose uptake [11]. Several other actions may contribute, such as increased intestinal use of glucose and decreased fatty acid oxidation [10]. Therefore, metformin can reduce peripheral insulin concentrations and improve glucose tolerance and metabolism. There are also preliminary in vitro data indicating that metformin may directly decrease ovarian androgen production [12]. Most studies have demonstrated the efficacy of metformin in inducing ovulation [13]-[15], whereas other reports disagree with these findings [16] [17]. Although metformin can be successful for unexplained infertility, women who are suffering for polycystic ovarian syndrome tend to have a positive response to metformin's ability to help the body respond better to insulin. Metformin can help make cycles more regular and increase fertility.

Gjonnaess reported an ovulation rate of $92 \%$ and a pregnancy rate of $70 \%$ after laparoscopic ovarian electrocoagulation in patients with PCOS [18]. This was the spark that reignited interest in surgical management of the disease and heralded the era of minimally invasive surgery in the treatment of these patients. Following this report, several studies described the success and utility of this procedure, with ovulation rates ranging from $64 \%$ to $92 \%$ and pregnancy rates from $41 \%$ to $80 \%$, [17] [19]-[24].

A meta-analysis of 13 randomized trials [25]-[27], comparing metformin with placebo, metformin plus CC, or CC alone in women with PCOS, concluded that metformin increased the ovulation rate by a factor of approximately four. The aim of the study was to compare the efficacy of metformin and laparoscopic ovarian drilling to be used in anovulatory patients with polycystic ovarian syndrome (PCOS) for ovulation induction and pregnancy achievement.

\section{Materials and Methods}

The male factor was normal in these two groups. All women in the study group were suffering from primary subfertility. An informed consent was taken from all the patients and the study was approved by the Scientific 
Counsel and Ethical committee of the institute. The patients who were newly diagnosed as PCOS and age less than 35 years old were included in the study group. Patients were excluded from the study if they were known to be diabetic or had underlying liver, renal, or heart disease, thyroid disorders or other hormone disorders, history of menstrual regulation (MR), induced abortion or dilation and curettage (D \& C), pelvic inflammatory disease (PID) and also those whose partner's sperm quality indicated male factor infertility on at least two occasions (sperm count $<10$ millions/mL, motility $<30 \%$, morphology $<30 \%$ ). Tubal patency was not tested before treatment. Pregnant patients were followed up until an ultrasound could document the viability of pregnancy. Patients randomized to the metformin arm were given the tablets at the initial dose of $500 \mathrm{mg}$ and increased in a stepwise fashion during the first 3 weeks to accommodate the side effects until patients were taking a total dose of $1500 \mathrm{mg} /$ day. With ovulation, the dose of drugs were unaltered in the group. Ovulation was detected either with a follicle diameter $>16 \mathrm{~mm}$ on transvaginal ultrasonography, or serum progesterone level. Serum progesterone (P) was done to assess the status of ovulation at day 0 and at day 120. If a woman ovulated, she continued taking the same dose of metformin until pregnancy was achieved. In females who became pregnant, metformin was continued for up to 12 weeks of pregnancy. In all groups urine pregnancy test was done 3 weeks after missed period. Pregnancy was confirmed by a gestational sac with fetal pole detection on ultrasonography. Laparoscopic Procedures: The patients included in this study were all operated upon by the author. Ovarian drilling was performed under general anaesthesia. The patient was put in Trendelenburg position. A subumbilical incision was made and and a $10-\mathrm{mm}$ trocar inserted intraperitoneally and pneumoperitoneum was created. The trocar sleeve was left in situ and a 10-mm 0 degree telescope was inserted and connected to a camera with a video monitor system. The abdominal cavity especially the pelvic area was carefully inspected. Two further 5-mm trocars were inserted through small incisions in each of the iliac fossae, one for a grasping forceps and the other for a unipolar diathermy electrode. The ovary was stabilized by grasping the ovarian ligament with a grasping forceps then 4 to 6 ovarian punctures was made to a depth of $6 \mathrm{~mm}$ using a unipolar electrode in both ovaries. Adequate care was taken to avoid aggressive drilling, which may lead to excessive tissue destruction, adhesion formation, and even ovarian failure. Diagnostic laparoscopy was performed in all patients. After a thorough assessment of the tubes, uterus, pelvis and liver in the upper abdomen, methylene blue hydrotubation was done to assess tubal patency.

\section{Statistical Analysis}

Data is expressed as mean \pm SD and evaluated statistically by using Student's paired t-test. For comparison of ovulation between Group I, Group II. Chi-square test is used \& P-value is calculated. SPSS version 14 was used for analysis.

\section{Results}

There were no significant differences in baseline variables in the study groups (Table 1) with regard to age, BMI and waist-to-hip ratio among the three groups. The mean age was 28.9 years and the mean BMI and waistto-hip ratio were 33.3 and 0.77 respectively. All the patients studied had morphological features of PCOs on TVS. The patients also had abnormal menstruation presenting as amenorrhea, which is defined as absent menstruation for more than 6 months, or oligomenorrhea, which is defined as intermenstual intervals of more than 35 days. In this study it was noted that hirsutism was not a main feature of patients diagnosed with PCOS.

Both groups had comparable proportions of primary subfertility and also duration of infertility was not significantly different among the study populations. There was also no significant difference regarding biochemical parameters, like FSH and LH, within the study groups (Table 2).

Baseline serum progesterone in two groups was very low indicating anovulation in these women but following administration of metformin and laparoscopic drilling serum progesterone was increased significantly from Day 1 to Day 120 with P-value of $<0.001$ in each group (Table 3) confirming ovulation in these patients.

The ovulation rate in metformin group and laparoscopy group was $68.4 \%$ and $59 \%$ respectively. The pregnancy rate (PR) was $20.1 \%$ in metformin group compared to $16.6 \%$ in laparoscopy group. The rate of spontaneous first trimester loss was three and two in metformin and laparoscopy group respectively. There were no ectopic pregnancies in metformin group and two in laparoscopy group. There were no second trimester pregnancy loss in metformin group, three in laparoscopy group. There were no multiple pregnancies in metformin group, two in laparoscopy group. The live birth per cycle in which ovulation occurred was higher in the metformin 
Table 1. Baseline characteristic of women with polycystic ovary syndrome.

\begin{tabular}{ccc}
\hline Characteristics & Metformin group & Laparoscopy group \\
Age, mean (SD) & $\mathrm{n}(152)$ & $\mathrm{n}(156)$ \\
\cline { 2 - 3 } WHR, mean (SD) & $29.6(4.35)$ & $0.77(0.14)$ \\
BMI, mean (SD) & $0.76(0.45)$ & $33.0(4.1)$ \\
Primary infertility, n (\%) & $32.9(4.2)$ & $136(89.5)$ \\
Length of infertility (y), mean (SD) & $112(71.8)$ & $3.3(0.14)$ \\
Morphology feature of PCO on U/S, n (\%) & $2.9(0.15)$ & $156(100)$ \\
Oligomenorrhea, n (\%) & $118(76.9)$ & $124(78.9)$ \\
Amenorrhea, $\mathrm{n}(\%)$ & $34(23.1)$ & $32(21.1)$ \\
\hline
\end{tabular}

Note: BMI: body mass index; WHR: waist-to-hip ratio.

Table 2. Baseline biochemical characteristics.

\begin{tabular}{cccc}
\hline & Metformin group & Laparoscopy group & P value \\
\cline { 2 - 3 } FSH (IU/L) & $\mathrm{n}(152)$ & $\mathrm{n}(156)$ & NS mean (SD) \\
LH (IU/L) & $4.5(1.95)$ & $12.9(1.75)$ & NS mean (SD) \\
\hline
\end{tabular}

Note: NS: not significant.

Table 3. Serum progesterone in metformin and laparoscopy groups at Day 0 and at Day 120.

\begin{tabular}{cccc}
\hline Serum progesterone (ng/ml) & Day 0 & Day 120 & P-value \\
\hline Metformin Group & $1.6 \pm 0.9$ & $11.3 \pm 6.8$ & $0.001^{* *}$ \\
Laparoscopy Group & $1.5 \pm 0.6$ & $10.3 \pm 4.7$ & $0.001^{* *}$ \\
\hline
\end{tabular}

*** Statistically highly significant.

group (18.4\%) than (15.4\%) in the laparoscopy groups, although the difference was not significant (Table 4).

Metformin at incremental doses was well tolerated and there were no drop outs in this study group. Three patients complained of nausea, dizziness and headache, but despite these side effects they continued through the study period. Metformin treatment also did not have any significant effect on biochemical parameters, particularly FSH, LH after 3 months of treatment and laparoscopy had the same effect as metformin (Table 5).

\section{Discussion}

One of the key aspects of achieving fertility is the process of ovulation by which an egg grows, matures and is ejected from the ovary on a regular (usually monthly) basis. Several medications like metformin, clomiphene citrate, letrozole are used by infertility specialists to enhance fertility and frequently they work by affecting ovulation. Surgical treatment in the form of wedge resection was the accepted treatment in polycystic ovarian syndrome. In the reported series the ovarian drilling resulted in ovulation in $90 \%$ of cases and conception in $70 \%$ of cases. Significant number of subsequent studies have produced similarly encouraging results. Laparoscopic ovarian drilling subsequently replaced the wedge resection as surgical treatment for women with PCO. Oral medications are often preferred as initial therapy due to their minimal expense, need for infrequent monitoring, and few side effects.

Our results demonstrated that ovulation was likely to occur after treatment with metformin and laparoscopy alone. In our study, the PR and live birth rate were slightly higher in the metformin group than laparoscopy group.

All of our patients had morphological features of PCO on ultrasound. Hsu et al. [28] noted a prevalence of PCO in $91 \%$ of the patients with PCOS. We tried to reduce the intra observer bias by assigning only two doctors 
Table 4. Rates of ovulation, pregnancy, pregnancy loss, and live birth.

\begin{tabular}{ccc}
\hline & Metformin & Laparoscopy \\
\cline { 2 - 3 } Ovulation & $\mathrm{n}(152)$ & $\mathrm{n}(156)$ \\
Pregnancy & $104 / 152(68.4 \%)$ & $92 / 156(59 \%)$ \\
First trimester loss & $32 / 152(20.1 \%)$ & $24 / 156(16.6 \%)$ \\
Ectopic & 3 & 2 \\
Second trimester loss & 0 & 3 \\
Multiple gestation & 0 & 2 \\
Live birth & 0 & $24 / 156(15.4 \%)$ \\
\hline
\end{tabular}

Table 5. Biochemical parameters at entry and after 3 months.

\begin{tabular}{ccccc}
\hline & \multicolumn{3}{c}{ LH (IU/L) } & FSH (IU/L) \\
\cline { 2 - 5 } & Initial & 3 months & Initial & 3 months \\
\hline Metformin & $11.3(5.41)$ & $11.7(5.10)$ & $4.5(1.95)$ & $4.3(2.0)$ \\
Laparoscopy & $12.2(2.95)$ & $11.2(3.45)$ & $4.9(1.75)$ & $5.0(1.9)$ \\
\hline
\end{tabular}

to do the TVS for the diagnosis of PCO. Metformin does not have an effect on the biochemical parameters of FSH, LH. This differs from some studies showing that metformin reduces the effect of LH and causes an elevation in serum FSH [29] [30] and agreed with studies showing that metformin has no effect on hormonal parameters [20] [31] [32].

Historically, the overall successful pregnancy rates after ovulation induction range from as low as $9 \%$ to as high as $25 \%$ [33]. In our study, consistent with the number of successful ovulatory cycles, pregnancy rate was notably higher in the metformin group compare to the laparoscopy group. Our results in this study are encouraging both in terms of the initiation and persistence of regular ovulatory cycles and in terms of pregnancies. It was reported that the beneficial effects of the surgical treatments are of limited duration, in most studies up to one year [26] [24].

\section{Conclusion}

For proper ovulation and conception not only an increase in serum progesterone is helpful but improvement in insulin resistance is required which is the key factor in the pathogenesis of PCOS. Our study showed that treatment with metformin, laparoscopy may be used as the first-line medications in anovulatory patients with polycystic ovarian syndrome (PCOS) for ovulation induction and pregnancy achievements. The study also demonstrated that metformin is superior to laparoscopy in inducing ovulation, pregnancy rate and live birth rate with polycystic ovarian syndrome. We could assert that laparoscopic ovarian drilling is an efficient treatment method for infertile anovulatory patients. The results obtained are as favourable as the biochemical profile of patients before surgery is less deteriorated. The costs incurred by the procedure can also recommend it as second-line therapy in comparison to metformin therapy, moreover laparoscopy is an invasive procedure.

\section{References}

[1] Frank, S. (1995) Polycystic Ovary Syndrome. New England Journal of Medicine, 333, 833-861.

[2] Stein, I.F. and Leventhal, M.L. (1934) Amenorrhea Associated with Bilateral Polycystic Ovaries. American Journal of Obstetrics \& Gynecology, 29, 181-191.

[3] Wild, R.A. (2002) Long-Term Health Consequences of PCOS. Human Reproduction Update, 8, 231-241. http://dx.doi.org/10.1093/humupd/8.3.231

[4] The Rotterdam ESHRE/ASRM-Sponsored PCOS Consensus Workshop Group (2004) Revised 2003 Consensus on Diagnostic Criteria and Long-Term Health Risks Related to Polycystic Ovary Syndrome. Human Reproduction, 19, 41-47.

[5] Hull, M.G. (1987) Epidemiology of Infertility and Polycystic Ovarian Disease: Endocrinological and Demographic 
Studies. Gynecological Endocrinology, 1, 235-245. http://dx.doi.org/10.3109/09513598709023610

[6] Homburg, R., Armar, N.A., Eshel, A., Adams, J. and Jacobs, H.S. (1988) Influence of Serum Luteinizing Hormone Concentrations on Ovulation, Conception and Early Pregnancy Loss in Polycystic Ovary Syndrome. BMJ, 297, 10241026. http://dx.doi.org/10.1136/bmj.297.6655.1024

[7] Boomsma, C.M., Eijkemans, M.J., Hughes, E.G., Visser, G.H., Fauser, B.C. and Macklon, N.S. (2006) A Meta-Analysis of Pregnancy Outcomes in Women with Polycystic Ovary Syndrome. Human Reproduction Update, 12, 673-683. http://dx.doi.org/10.1093/humupd/dml036

[8] Kim, H., Taylor, A.E. and Barbieri, R.L. (2000) Insulin Sensitizers and Polycystic Ovary Syndrome: Can a Diabetes Medication Treat Infertility? Fertility and Sterility, 73, 1097-1098. http://dx.doi.org/10.1016/S0015-0282(00)00540-9

[9] Velazquez, E.M., Mendoza, S., Hamer, T., Sosa, F. and Glueck, C.J. (1994) Metformin Therapy in Polycystic Ovary Syndrome Reduces Hyperinsulinemia, Insulin Resistance, Hyperandrogenemia, and Systolic Blood Pressure, While Facilitating Normal Menses and Pregnancy. Metabolism, 43, 647-654. http://dx.doi.org/10.1016/0026-0495(94)90209-7

[10] Bailey, C.S. and Turner, R.C. (1996) Metformin Drug Therapy. New England Journal of Medicine, 334, 574-579. http://dx.doi.org/10.1056/NEJM199602293340906

[11] De Fronzo, R.A., Barzilai, N. and Simonson, D.C. (1991) Mechanism of Metformin Action in Obese and Lean Noninsulin-Dependent Diabetic Subjects. Journal of Clinical Endocrinology and Metabolism, 73, 1294-1301. http://dx.doi.org/10.1210/jcem-73-6-1294

[12] Attia, G.R., Rainey, W.E. and Carr, B.R. (2001) Metformin Directly Inhibits Androgen Production in Human Theca Cells. Fertility and Sterility, 76, 517-524. http://dx.doi.org/10.1016/S0015-0282(01)01975-6

[13] Nestler, J.E., Jakubowicz, D.J., Evans, W.S. and Pasquali, R. (1998) Effects of Metformin on Spontaneous and Clomiphene Induced Ovulation in the Polycystic Ovary Syndrome. New England Journal of Medicine, 338, 1876-1880. http://dx.doi.org/10.1056/NEJM199806253382603

[14] Velazquez, E.M., Acosta, A. and Mendoza, S.G. (1997) Menstrual Cyclicity after Metformin Therapy in Polycystic Ovary Syndrome. Obstetrics \& Gynecology, 90, 392-395. http://dx.doi.org/10.1016/S0029-7844(97)00296-2

[15] Moghetti, P., Castello, R., Negri, C., Tosi, F., Perrone, F., Caputo, M., et al. (2000) Metformin Effects on Clinical Features, Endocrine and Metabolic Profiles, and Insulin Sensitivity in Polycystic Ovary Syndrome: A Randomized, Double-Blind, Placebo-Controlled 6 Month Trial, Followed by Open, Long-Term Clinical Evaluation. Journal of Clinical Endocrinology and Metabolism, 85, 139-146.

[16] Ehrmann, D.A., Cavaghan, M.K., Imperial, J., Sturis, J., Rosenfield, R.L. and Polonsky, K.S. (1997) Effects of Metformin on Insulin Secretion, Insulin Action, and Ovarian Steroidogenesis in Women with Polycystic Ovary Syndrome. Journal of Clinical Endocrinology and Metabolism, 82, 524-530.

[17] Sahin, Y., Yirmibeş, U., Keleştimur, F. and Aygen, E. (2004) The Effects of Metformin on Insulin Resistance, Clomiphene-Induced Ovulation and Pregnancy Rates in Women with Polycystic Ovary Syndrome. European Journal of Obstetrics, Gynecology and Reproductive Biology, 113, 214-220.

[18] Norman, R.J. (2004) Metformin—Comparison with Other Therapies in Ovulation Induction in Polycystic Ovary Syndrome. Journal of Clinical Endocrinology \& Metabolism, 89, 4797-4800. http://dx.doi.org/10.1210/jc.2004-1658

[19] Badawy, A., Mosbah, A., Tharwat, A. and Eid, M. (2009) Extended Letrozole Therapy for Ovulation Induction in Clomiphene-Resistant Women with Polycystic Ovary Syndrome: A Novel Protocol. Fertility and Sterility, 92, 236239. http://dx.doi.org/10.1016/j.fertnstert.2008.04.065

[20] Kar, S. (2012) Clomiphene Citrate or Letrozole as First-Line Ovulation Induction Drug in Infertile PCOS Women: A Prospective Randomized Trial. Journal of Human Reproductive Sciences, 5, 262-265. http://dx.doi.org/10.4103/0974-1208.106338

[21] Elizur, S.E. and Tulandi, T. (2008) Drugs in Infertility and Fetal Safety. Fertility and Sterility, 89, 1595-1602. http://dx.doi.org/10.1016/j.fertnstert.2008.02.092

[22] Buttram Jr., V.C. and Vacquero, C. (1975) Postovarian Wedge Resection Adhesive Disease. Fertility and Sterility, 26, 874-876.

[23] Gjonnaess, H. (1984) Polycystic Ovarian Syndrome Treated by Ovarian Electrocautery through the Laparoscope. Fertility and Sterility, 41, 20-25.

[24] Keckstein, J. (1989) Laparoscopic Treatment of Polycystic Ovarian Syndrome in Laparoscopic Surgery. Baillière’s Clinical Obstetrics and Gynaecology, 3, 563-581. http://dx.doi.org/10.1016/S0950-3552(89)80009-4

[25] Lord, J.M., Flight, I.H.K. and Norman, R.J. (2003) Metformin in Polycystic Ovary Syndrome: Systematic Review and Meta-Analysis. British Medical Journal, 327, 951-953. http://dx.doi.org/10.1136/bmj.327.7421.951

[26] Lord, J.M., Flight, I.H.K. and Norman, R.J. (2003) Insulin-Sensitising Drugs (Metformin, Troglitazone, Rosiglitazone, 
Pioglitazone, D-Chiro-Inositol) for Polycystic Ovarian Syndrome. Cochrane Database of Systematic Reviews, 2003, Article ID: CD003053.

[27] Mitwally, M.F.M. and Casper, R.F. (2002) Aromatase Inhibition for Ovarian Stimulation: Future Avenues for Infertility Management. Current Opinion in Obstetrics \& Gynecology, 14, 255-263. http://dx.doi.org/10.1097/00001703-200206000-00003

[28] Hsu, M.I., Liou, T.H., Chou, S.Y., Chang, C.Y. and Hsu, C.S. (2007) Diagnostic Criteria for Polycystic Ovary Syndrome in Taiwanese Chinese Women: Comparison between Rotterdam 2003 and NIH 1990. Fertility and Sterility, 88, 727-729. http://dx.doi.org/10.1016/j.fertnstert.2006.11.149

[29] Aruna, J., Mittal, S., Kumar, S., Misra, R., Dadhwal, V. and Vimala, N. (2004) Metformin Therapy in Women with Polycystic Ovary Syndrome. International Journal of Gynaecology and Obstetrics, 87, 237-241.

[30] Sugimoto, O. and Aono (1993) The Committee for Reproductive and Endocrine in Japan Society of Obstetrics and Gynaecology. Annual Report (1991-1992) for the Determination of Diagnostic Criteria for PCOS. Acta Obstetrica et Gynaecologica Japonica, 45, 1359-1367.

[31] Nestler, J.E. and Jakubowicz, D.J. (1996) Decreases in Ovarian Cytochrome P450c17 $\alpha$ Activity and Serum Free Testosterone after Reduction of Insulin Secretion in Polycystic Ovary Syndrome. New England Journal of Medicine, 335, 617-623. http://dx.doi.org/10.1056/NEJM199608293350902

[32] Fleming, R., Hopkinson, Z.E., Wallace, A.M., Greer, I.A. and Sattar, N. (2002) Ovarian Function and Metabolic Factors in Women with Oligomenorrhea Treated with Metformin in a Randomized Double Blind Placebo-Controlled Trial. Journal of Clinical Endocrinology \& Metabolism, 87, 569-574. http://dx.doi.org/10.1210/jcem.87.2.8261

[33] Balen, A. (2000) Ovulation Induction for Polycystic Ovary Syndrome. Fertility and Sterility, 3, 106-111. 